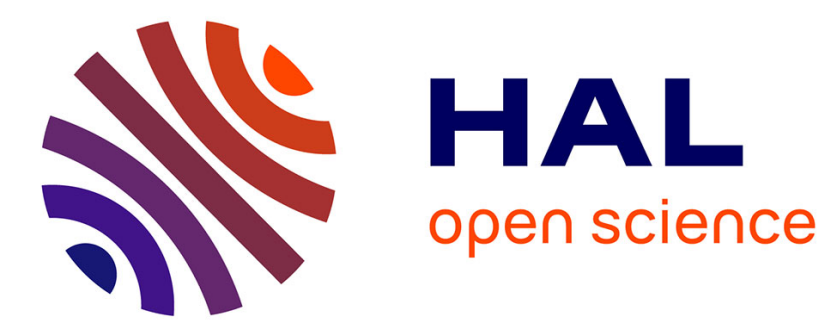

\title{
Robust shortest path problems
}

\author{
Virginie Gabrel, Cecile Murat
}

\section{- To cite this version:}

Virginie Gabrel, Cecile Murat. Robust shortest path problems. 2007. hal-00179975

\section{HAL Id: hal-00179975 \\ https://hal.science/hal-00179975}

Preprint submitted on 17 Oct 2007

HAL is a multi-disciplinary open access archive for the deposit and dissemination of scientific research documents, whether they are published or not. The documents may come from teaching and research institutions in France or abroad, or from public or private research centers.
L'archive ouverte pluridisciplinaire HAL, est destinée au dépôt et à la diffusion de documents scientifiques de niveau recherche, publiés ou non, émanant des établissements d'enseignement et de recherche français ou étrangers, des laboratoires publics ou privés. 


\title{
Robust shortest path problems
}

\author{
Virginie Gabrel*, Cécile Murat*
}

\begin{abstract}
Résumé
Cet article constitue un état de l'art sur les problèmes de plus courts chemins pour lesquels il existe des éléments d'incertitude et d'indétermination sur les valeurs des arcs. Deux modèles d'incertitude sont distingués : celui dit par intervalle et celui dit par scénarios. Différentes mesures et approches de la robustesse sont présentées : celles issues de la théorie de la décison, celles issues de l'analyse multicritère et celles issues de la programmation mathématique. Elles donnent lieu à plusieurs versions distinctes du problème de plus court chemin robuste dont les complexités et les résolutions sont présentées.
\end{abstract}

Mots-clefs : plus court chemin, optimisation robuste, incertitudes sur les données, scénario du pire cas, regret maximum

\begin{abstract}
This paper is a state of the art on the shortest path problems for which it exists uncertainty and inaccuracy factors on arc values. Two uncertainty models are distinguished: the so-called interval model and the discrete set of scenarios model. Different measures and approaches of robustness are presented: those coming from decision theory, those coming from multicriteria analysis and those coming from mathematical programming. Each one leads to a particular version of robust shortest path problem for which complexity and resolution are studied.
\end{abstract}

Key words : shortest path, robust optimization, data uncertainties, worst case scenario, maximal regret

*LAMSADE, Université Paris-Dauphine, 75775 Paris cedex 16, France. \{gabrel, murat \} @lamsade. dauphine.fr 


\section{Introduction}

In optimization, it is used to deal with uncertain and inaccurate factors which make difficult the assignment of a single plausible value to each model parameters. Two approaches are possible : in the first one, a single nominal value is assigned to each parameter, the corresponding optimal solution is computed, then the interval in which each parameter can vary in order to preserve optimality solution is determined ; the second approach consists in taking into account in the model to optimize, the possible variations of each parameter. In mathematical programming, the first approach is known as sensibility analysis. For the second approach, stochastic optimization may be applied for some problems in which parameters' value can be described by probability laws. When it is not possible nor relevant to associate probability laws to parameters, another way amounts to assign a set of possible values to each parameter. In this context, the choice of one value in each set corresponds to a scenario. The induced robust optimization problem is to determine a single solution which is optimal for all scenarios. In general, such a solution does not exist and the problem is to determine a "relatively good" solution for all scenarios.

In most of robustness studies in combinatorial optimization, a robust solution is a solution which is acceptable in a large majority of scenarios and, which is never too bad (cf for example $[23,25])$. This characterization leaves place to many possible interpretations and, thus gives place to various approaches of robustness. These approaches differ from models used to represent the uncertain and inaccurate factors of the considered decision problem, from methodology used to measure robustness, and finally from the analysis and the design of resolution methods.

In this chapter, we present various models, criteria and methods which were studied for the robust shortest path problem. In the section 2, models are proposed then comes in the section 3, the presentation of different measures of robustness, and finally, in the parts 4 and 5, is presented complexities and resolutions of various versions of the robust shortest path problem.

\section{Models for representing uncertain, undetermined and random factors}

We consider a directed valued graph $G=(X, U)$ such as $X=\{1, \ldots, n\}$ and $|U|=$ $m$. A path $\mu$ from 1 to $n$ in $G$ is a sequence of arcs : $\mu=\left\{\left(i_{0}=1, i_{1}\right),\left(i_{1}, i_{2}\right), \ldots,\left(i_{k-1}, i_{k}=\right.\right.$ $n)\}$. Within the deterministic framework, to each arc $u=(i, j) \in U$ is associated a single value $c_{u}=c_{i j}$. The value of $\mu$ is then given by : $c(\mu)=\sum_{(i, j) \in \mu} c_{i j}$. A traditional problem of combinatorial optimization consists in determining the shortest path, denoted by $\mu_{G}^{*}$, among the set $\mathcal{C}$ of paths from 1 to $n$ in $G$. 
When elements of uncertainty and/or inaccuracy must be taken into account, it can be no more relevant, even possible, to set a single value $c_{i j}$ for each arc $(i, j)$.

In some contexts, these values are described by probability laws. The problem then becomes the stochastic shortest path problem that we will not study in this paper.

In other contexts, uncertain, inaccurate and random factors cannot be efficiently modeled by probability laws. Since the middle of the 90', two models were more particularly developed in the literature : the so-called interval model and the discrete set of scenarios model. In these two models, each arc is associated with a set of values, this set may be of infinite or finite size. The assumption is that on each arc, the real value belongs to its associated set. A scenario corresponds to the specification of a single value for each arc. We will note $S$ the set of $q$ relevant scenarios, $c_{i j}^{s}$ the value of $\operatorname{arc}(i, j)$ under scenario $s$, $c^{s}(\mu)$ the value of path $\mu$ under scenario $s, G^{s}$ the graph valued according to the scenario $s, \mu_{G^{s}}^{*}$ the shortest path from 1 to $n$ in $G^{s}$.

In the two next sections, we present the so-called interval model and the so-called discrete set of scenarios model.

\subsection{The interval model}

In the interval model, each arc $(i, j)$ is associated to an interval, denoted $\left[b_{i j}, B_{i j}\right]$, such as $c_{i j} \in\left[b_{i j}, B_{i j}\right]$. This interval represents the set of possible values of the arc $(i, j)$. The set $S$ of scenarios is infinite and is defined as the cartesian product of the $m$ intervals $\left[b_{u}, B_{u}\right]$. In this context, a path $\mu$ can take any value belonging to the interval $[b(\mu), B(\mu)]$ with $b(\mu)=\sum_{(i, j) \in \mu} b_{i j}$ and $B(\mu)=\sum_{(i, j) \in \mu} B_{i j}$.

EXAMPLE. Let us consider the path $\mu=(1,2,4,5,7)$ in the graph $G$ of the figure 1 whose arcs are valued by intervals. Under any scenario $s$, this path has a value $c^{s}(\mu)$ belonging to the interval $[5,24]$.

This model is considered for the shortest path problem in $[4,13,14,17,19,18,28]$.

In addition, the interval model is applied to other combinatorial problems, for example the minimum spanning tree $[16,26]$, the problem of selecting $p$ elements among $n$ with minimal total weight [3], or the assignment problem [1].

\subsection{The discrete set of scenarios model}

In the discrete set of scenarios model, a finite set $S$ of scenarios is considered. Thus, given $q$ scenarios, each arc $(i, j)$ is associated to a finite set of $q$ plausible values $\left\{c_{i j}^{1}, \ldots, c_{i j}^{q}\right\}$. 


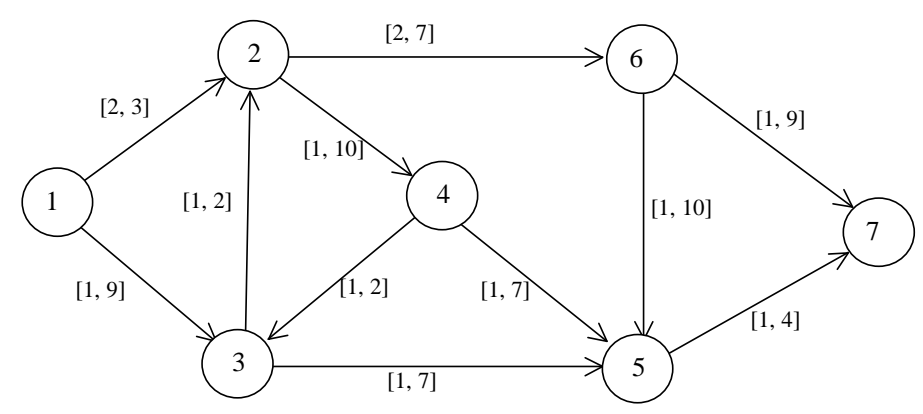

FIG. 1 - Example of interval model

Let us note that on the contrary of interval model, any combination of values $c_{i j}$ does not constitute a relevant scenario. Consequently, each path $\mu$ is associated to a $q$ dimension vector $V(\mu)$ such as $V(\mu)=\left(c^{1}(\mu), \ldots, c^{q}(\mu)\right)$, where $c^{s}(\mu)=\sum_{(i, j) \in \mu} c_{i j}^{s}$ with $s \in\{1, \ldots, q\}$.

EXAMPLE. Let us consider the graph $G$ of the figure 2 whose arcs are valued according to three scenarios.

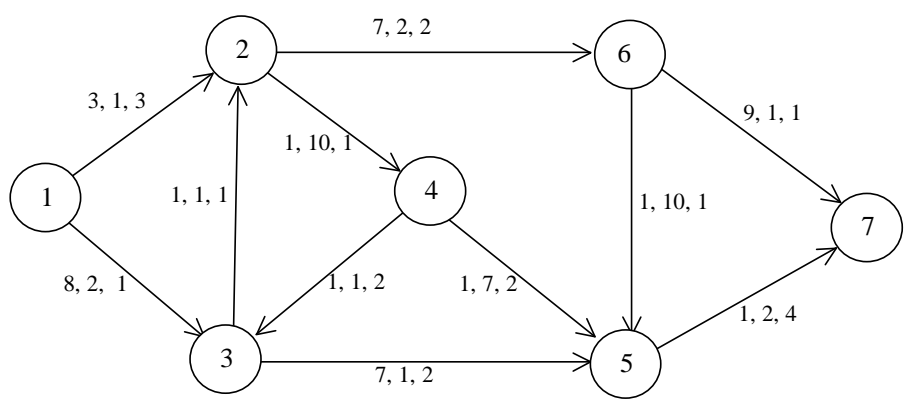

FIG. 2 - Example of scenarios model

Elementary paths from 1 to 7 and their values on the three scenarios are presented in the table 1 .

This model is considered for the shortest path problem in [15, 21, 24, 27].

In addition, the discrete set of scenarios model is applied to other combinatorial problems, for example the minimum spanning tree in $[15,24]$, the problem of selecting $p$ elements among $n$ with minimal total weight in [3], the 1-median location problem in 


\begin{tabular}{|c||c|c|c|}
\hline$\mu$ & $c^{1}(\mu)$ & $c^{2}(\mu)$ & $c^{3}(\mu)$ \\
\hline \hline$\mu_{1}=(1,2,6,7)$ & 19 & 4 & 6 \\
$\mu_{2}=(1,2,6,5,7)$ & 12 & 15 & 10 \\
$\mu_{3}=(1,2,4,5,7)$ & 6 & 20 & 10 \\
$\mu_{4}=(1,2,4,3,5,7)$ & 13 & 15 & 12 \\
$\mu_{5}=(1,3,2,6,7)$ & 25 & 6 & 5 \\
$\mu_{6}=(1,3,2,4,5,7)$ & 12 & 22 & 9 \\
$\mu_{7}=(1,3,2,6,5,7)$ & 18 & 17 & 9 \\
$\mu_{8}=(1,3,5,7)$ & 16 & 5 & 7 \\
\hline
\end{tabular}

ТАВ. 1 - Elementary paths from 1 to 7

$[12,15]$, or the assignment problem $[1,7]$.

A more general model is presented in [8] which includes the two previous models. In this general framework, arcs value are determined by a set of $r$ parameters. These parameters are not exactly known and two models are considered : a discrete set of parameters $r$-tuples, and a continuous subset of $R^{r}$ delimited by constraints (for which the interval model is a particular case).

\section{Robustness measures}

In this section, we list models to evaluate a path in term of robustness. Three approaches can be distinguished : the first one, coming from the decision theory, models the concept of robustness through a single criterion to be optimized; the second one uses the mathematical programming to address data uncertainty; and the third one, coming from the multicriteria analysis and the social choice theory, attempts to characterize the robust solutions by a set of conditions to be checked.

\subsection{Classical criteria resulting from the decision theory}

\subsubsection{Worst case criterion}

Given a path, the scenario to be considered is the one that gives the worst value for this path. In this context, the value of a path $\mu$, noted $v_{\mathrm{woR}}(\mu)$, is defined by:

$$
v_{\mathrm{WOR}}(\mu)=\max _{s \in S} c^{s}(\mu)
$$


EXAMPLE. Let us consider the path $\mu=(1,2,4,5,7)$ of the graph in the figure 1 . The worst case scenario is the one for which each arc value is equal to its upper bound. In this case, $v_{\mathrm{WOR}}(\mu)=24$.

The problem is to determine the path $\mu_{\mathrm{WOR}}^{*}$ which minimizes $v_{\mathrm{WOR}}$ as follows :

$$
v_{\mathrm{WOR}}\left(\mu_{\mathrm{WOR}}^{*}\right)=\min _{\mu \in \mathcal{C}} v_{\mathrm{WOR}}(\mu)
$$

Under any scenario $s \in S, \mu_{\mathrm{wOR}}^{*}$ has a value lower or equal to $v_{\mathrm{WOR}}\left(\mu_{\mathrm{wOR}}^{*}\right)$. Thus, $v_{\mathrm{wOR}}\left(\mu_{\mathrm{wOR}}^{*}\right)$ can be considered as an upper bound which offers an absolute guarantee. That is why in the work of Kouvelis and Yu [15], solutions which optimize this criterion are called absolute robust solutions. In decision theory, this criterion models the behavior of a decision maker averse to risk.

\subsubsection{The maximum regret criterion}

Given a scenario $s$, the choice of a path $\mu$ which is not necessarily a shortest path in $G^{s}$, generates a regret denoted $r^{s}(\mu)=c^{s}(\mu)-c^{s}\left(\mu_{G^{s}}^{*}\right)$. A path $\mu$ is then evaluated by $v_{\mathrm{REG}}(\mu)$ on the basis of the maximum regret scenario :

$$
v_{\mathrm{REG}}(\mu)=\max _{s \in S} r^{s}(\mu)
$$

EXAMPLE. Let us consider the path $\mu=(1,2,4,5,7)$ of the graph in the figure 1 and the scenario $s^{b}$ in which each arc value of $U$ is set to its lower bound. Then, as the shortest path of $G^{s^{b}}$ is $\mu_{G^{s}}^{*}=(1,3,5,7)$, we have $: r^{s^{b}}(\mu)=5-3=2$. In fact, we will see in the section 4.2 that the maximum regret associated to $\mu$ is of 19 , and thus : $v_{\mathrm{REG}}(\mu)=19$.

The optimal solution according to the maximum regret criterion will be denoted $\mu_{\mathrm{REG}}^{*}$ and checks :

$$
v_{\mathrm{REG}}\left(\mu_{\mathrm{REG}}^{*}\right)=\min _{\mu \in \mathcal{C}} v_{\mathrm{REG}}(\mu)
$$

EXAMPLE. Let us consider again the graph in the figure 2 for which three scenarios are taking into account. Since the shortest path in each scenario is respectively $\mu_{G^{1}}^{*}=$ $(1,2,4,5,7)$ of value $6, \mu_{G^{2}}^{*}=(1,2,6,7)$ of value 4 and $\mu_{G^{3}}^{*}=(1,3,2,6,7)$ of value 5 , we can determine the values $v_{\mathrm{WOR}}(\mu)$ and $v_{\mathrm{REG}}(\mu)$ for any path $\mu$, presented in the table 2 .

Optimal solutions according to the worst case criterion are paths $\mu_{2}$ and $\mu_{4}$, and the optimal solution according to the maximum regret criterion is the path $\mu_{8}$.

In the work of Kouvelis and Yu [15], a solution which optimizes the maximum regret criterion is called a robust deviation solution. In addition, they propose another criterion of 


\begin{tabular}{|c||c|c|}
\hline$\mu$ & $v_{\mathrm{WOR}}(\mu)$ & $v_{\mathrm{REG}}(\mu)$ \\
\hline \hline$\mu_{1}=(1,2,6,7)$ & 19 & 13 \\
$\mu_{2}=(1,2,6,5,7)$ & 15 & 11 \\
$\mu_{3}=(1,2,4,5,7)$ & 20 & 16 \\
$\mu_{4}=(1,2,4,3,5,7)$ & 15 & 11 \\
$\mu_{5}=(1,3,2,6,7)$ & 25 & 19 \\
$\mu_{6}=(1,3,2,4,5,7)$ & 22 & 18 \\
$\mu_{7}=(1,3,2,6,5,7)$ & 18 & 13 \\
$\mu_{8}=(1,3,5,7)$ & 16 & 10 \\
\hline
\end{tabular}

TAB. 2 - Paths value on classical criteria

robustness in term of relative deviation which consists in normalizing the regret measure as follows :

$$
v_{\mathrm{REG}^{\prime}}(\mu)=\max _{s \in S} \frac{c^{s}(\mu)-c^{s}\left(\mu_{G^{s}}^{*}\right)}{c^{s}\left(\mu_{G^{s}}^{*}\right)}
$$

This criterion must also be minimized.

Classical criteria coming from decision theory evaluate solutions on the basis of extreme scenarios. The major disadvantage is to focus on a single scenario, mainly the worst case, without taking into account all others scenarios. On the other hand, these criteria offer optimality guarantees. In particular, Averbakh in [2] proposes a very lighting interpretation of the maximum regret criterion. He supposes, rather naturally, that a solution is robust if it is a good solution on all the scenarios. Thus, for a scenario $s$, robust solutions must belong to the set, denoted $\mathcal{C}_{\epsilon}^{s}$, of the $\epsilon$-optimal solutions verifying :

$$
c^{s}(\mu)-c^{s}\left(\mu_{G^{s}}^{*}\right) \leq \epsilon .
$$

The set, denoted $\mathcal{C}_{\epsilon}^{\mathrm{ROB}}$, of robust solutions is then the intersection of all $\epsilon$-optimal solutions sets, one per scenario, as follows :

$$
\mathcal{C}_{\epsilon}^{\mathrm{ROB}}=\bigcap_{s \in S} \mathcal{C}_{\epsilon}^{s}
$$

It is obvious that if $\epsilon$ is too small, $\mathcal{C}_{\epsilon}^{\mathrm{ROB}}$ will be empty, and the cardinality of $\mathcal{C}_{\epsilon}^{\mathrm{ROB}}$ increases with $\epsilon$. Otherwise, if $\epsilon$ is too large, then $\mathcal{C}_{\epsilon}^{\mathrm{ROB}}$ will contain solutions with bad evaluations on some scenarios. Consequently, it is particularly interesting to determine the smallest value of $\epsilon$ such as $\mathcal{C}_{\epsilon}^{\mathrm{ROB}}$ contains at least one solution. Averbakh shows that this value is not other than $v_{\mathrm{REG}}\left(\mu_{\mathrm{REG}}^{*}\right)$. Indeed, solutions of $\mathcal{C}_{\epsilon}^{\mathrm{ROB}}$ are such that :

$$
\begin{aligned}
\forall \mu \in \mathcal{C}_{\epsilon}^{\mathrm{ROB}}, \quad \forall s \in S, c^{s}(\mu)-c^{s}\left(\mu_{G^{s}}^{*}\right) \leq \epsilon \\
\Rightarrow \quad \forall \mu \in \mathcal{C}_{\epsilon}^{\mathrm{ROB}}, \quad \max _{s \in S}\left(c^{s}(\mu)-c^{s}\left(\mu_{G^{s}}^{*}\right)\right)=v_{\mathrm{REG}}(\mu) \leq \epsilon
\end{aligned}
$$


But, since $\forall \mu \in \mathcal{C}_{\epsilon}^{\mathrm{ROB}}, v_{\mathrm{REG}}(\mu) \geq v_{\mathrm{REG}}\left(\mu_{\mathrm{REG}}^{*}\right)$, if $\epsilon<v_{\mathrm{REG}}\left(\mu_{\mathrm{REG}}^{*}\right)$ then $\mathcal{C}_{\epsilon}^{\mathrm{ROB}}$ is empty, and otherwise, $\mathcal{C}_{\epsilon}^{\mathrm{ROB}}$ contains at least $\mu_{\mathrm{REG}}^{*}$. Consequently, $v_{\mathrm{REG}}\left(\mu_{\mathrm{REG}}^{*}\right)$ is the smallest $\epsilon$ such as it exists at least one solution which is $\epsilon$-optimal for all scenarios. This interpretation may be adapted to the relative deviation criterion by modifying the definition of $\epsilon$-optimal solution as follows : for a scenario $s, \mu$ is $\epsilon$-optimal if and only if $\frac{c^{s}(\mu)-c^{s}\left(\mu_{G^{s}}^{*}\right)}{c^{s}\left(\mu_{G^{s}}^{*}\right)} \leq \epsilon$.

\subsection{Methodology coming from mathematical programming}

The shortest path problem can be represented by an integer linear program of the form :

$$
(\mathcal{P}) \begin{cases}\min & \sum_{(i, j) \in U} c_{i j} y_{i j} \\ \text { s.c } & y \in Y\end{cases}
$$

In studies dealing with robustness in mathematical programming, we can distinguish those in which data uncertainty affects only the elements of the constraint matrix, and those in which data uncertainty concerns only objective function coefficients. In this context, a very interesting approach is introduced by Bertsimas and Sim (in [5] and [6]). They consider the following interval model for objective function coefficients : $c_{i j} \in\left[\bar{c}_{i j}, \bar{c}_{i j}+\widehat{c}_{i j}\right]$ where $\bar{c}_{i j}$ is the nominal value for $(i, j)$ and $\widehat{c}_{i j} \geq 0$ represents the deviation from the nominal coefficient $\bar{c}_{i j}$. They start from the quite natural idea that the worst case will not happen simultaneously for all coefficients. In [5], authors "stipulate that nature will be restricted in its behavior, in that only a subset of coefficients will change in order to adversely affect the solution".

So, they introduce a parameter $\Gamma$ which represents the maximum number of coefficients that can deviate from their nominal value $: \Gamma=0$ means that none coefficient will vary, while $\Gamma=m$ means that all coefficients will vary in the worst case sense. So, $\Gamma$ is interpreted as a level of robustness.

The robust version of the shortest path problem becomes :

$$
\left(\mathcal{P}_{\mathrm{ROB}}\right)\left\{\begin{array}{l}
\min \left(\sum_{(i, j) \in U} \bar{c}_{i j} y_{i j}+\max _{\{R|R \subseteq U,| R \mid \leq \Gamma\}} \sum_{(i j) \in R} \widehat{c}_{i j} y_{i j}\right) \\
\text { s.c } y \in Y
\end{array}\right.
$$

\subsection{Methodology coming from multicriteria analysis}

In multicriteria analysis [22], a decision problem is commonly defined using a set of solutions, a discrete set of criteria and an aggregation model of these criteria. A solution 
is thus described by its evaluation vector containing the solution value on each criterion. Considering a scenario as a criterion, a part of the results obtained in multicriteria analysis can be used for the robustness analysis (cf [11]). In this context, the robustness analysis is based upon the choice of an evaluation vector associated with each solution, and the definition of an aggregation model of these evaluation vectors.

\subsubsection{Path evaluation vector}

When the set of scenarios is discrete, it is natural to consider the vector $V(\mu)$, representing all possible values of $\mu$ on various scenarios, as an evaluation vector (cf [8] and [11]).

More recent work ([12] and [21]) applies the anonymous principle which consists in admitting that the vector $V(\mu)=\left(c^{1}(\mu), \ldots, c^{q}(\mu)\right)$ is equivalent to the vector $V(\mu)=$ $\left(c^{\sigma(1)}(\mu), \ldots, c^{\sigma(q)}(\mu)\right)$ for any permutation $\sigma(i)$ of the $q$ scenarios. According to this principle, a path $\mu$ can be evaluated by a vector obtained by ordering the values $c^{i}(\mu)$ in a decreasing order. This vector is called disutility vector in [12], and is denoted $D(\mu)=$ $\left(d^{1}(\mu), \ldots, d^{q}(\mu)\right)$, where $d^{1}(\mu) \geq \ldots \geq d^{q}(\mu)$ with $d^{i}(\mu)$ representing the value of order $i$ of the path $\mu$.

In some other studies on robustness, another so-called equity principle is proposed. According to this principle, a solution whose evaluations are well distributed around the average on the various scenarios is always preferred to a more unbalanced solution. For that, one evaluates a solution on the basis of the following vector, called in [21] the generalized Lorenz vector : $L(\mu)=\left(l^{1}(\mu), \ldots, l^{q}(\mu)\right)$ with $l^{j}(\mu)=\sum_{i=1}^{j} d^{i}(\mu)$.

EXAMPLE. For the graph in figure 2, desutility and generalized Lorenz vectors are presented for each path in the table 3 .

\begin{tabular}{|c||c|c|c||c|c|c|}
\hline \multicolumn{1}{|c||}{} & \multicolumn{3}{c||}{ Desutility vectors } & \multicolumn{3}{c|}{ Lorenz vectors } \\
\hline$\mu$ & $d^{1}(\mu)$ & $d^{2}(\mu)$ & $d^{3}(\mu)$ & $l^{1}(\mu)$ & $l^{2}(\mu)$ & $l^{3}(\mu)$ \\
\hline$\mu_{1}=(1,2,6,7)$ & 19 & 6 & 4 & 19 & 25 & 29 \\
$\mu_{2}=(1,2,6,5,7)$ & 15 & 12 & 10 & 15 & 27 & 37 \\
$\mu_{3}=(1,2,4,5,7)$ & 20 & 10 & 6 & 20 & 30 & 36 \\
$\mu_{4}=(1,2,4,3,5,7)$ & 15 & 13 & 12 & 15 & 28 & 40 \\
$\mu_{5}=(1,3,2,6,7)$ & 25 & 6 & 5 & 25 & 31 & 36 \\
$\mu_{6}=(1,3,2,4,5,7)$ & 22 & 12 & 9 & 22 & 34 & 43 \\
$\mu_{7}=(1,3,2,6,5,7)$ & 18 & 17 & 9 & 18 & 35 & 44 \\
$\mu_{8}=(1,3,5,7)$ & 16 & 7 & 5 & 16 & 23 & 28 \\
\hline
\end{tabular}

ТАВ. 3 - Evaluation vectors for paths from 1 to 7 


\subsubsection{Aggregation models for robustness}

In multicriteria analysis, the dominance relation is defined as follows.

DEFINITION. Given a family $F=\left(f^{1}, \ldots, f^{q}\right)$ of $q$ criteria, a path $\mu$ dominates a path $\mu^{\prime}$ if and only if $f^{i}(\mu) \leq f^{i}\left(\mu^{\prime}\right)$ for $i=1, \ldots, q$, with at least one strict inequality. The path $\mu^{\prime}$ is then dominated by $\mu$, that one notes $\mu \Delta_{F} \mu^{\prime}$.

Definition. A path $\mu$ is non dominated if and only if there is not another path $\mu^{\prime} \in \mathcal{C}$ such as $\mu^{\prime} \Delta_{F} \mu$.

In the interval model, it is not possible to associate to each path a finite number of evaluation vectors because the number of scenarios is infinite. However, dominance relation remains defined in this model as follows

Definition. A path $\mu$ dominates a path $\mu^{\prime}$ if and only if $c^{s}(\mu) \leq c^{s}\left(\mu^{\prime}\right)$ for all $s \in S$, with at least one strict inequality. The path $\mu^{\prime}$ is then dominated by $\mu$ that one notes $\mu \Delta \mu^{\prime}$.

Whatever the evaluation vector, robust solutions naturally belong to the non-dominated solutions set. Thus, the problem can be first to determine all non-dominated solutions (see for example [8]).

EXAMPLE. For the graph in figure 2, and regarding to the evaluation vector $V(\mu)$ (in table 1), all solutions are non-dominated excepted $\mu_{4}$ which is dominated by $\mu_{2}$; regarding to the desutility vector (in table 3 ), non-dominated solutions are $\mu_{1}, \mu_{2}, \mu_{8}$; and regarding to the Lorenz vector, non-dominated solutions are $\mu_{2}$ and $\mu_{8}$. Let us remark that the path $\mu_{4}$ which is optimal for the worst case criterion is a dominated path.

The main difficulty comes from the possible huge number of non-dominated paths. Hansen in [10] has defined a bicriteria graph for which the number of non-dominated paths is an exponential function of the graph order. Perny and Spanjaard showed in [21] that the non-dominated solutions set according to Lorenz evaluation vectors is a subset of the non-dominated solutions set according to $V(\mu)$ vectors. Thus, in the graph proposed by Hansen, only two paths remain non-dominated according to Lorenz evaluation vectors. However, in [21], a graph with an exponential number of non-dominated paths according to Lorenz evaluation vectors is proposed.

Consequently, the determination of all non-dominated solutions is not possible in general case. Other procedures must be defined for aggregating evaluation vectors in order to provide one or a small size set of robust solutions. Let us mention an alternative approach based on a lexicographic procedure. This approach is applied in [12] on the disutility 
vector for the 1-median location problem.

These three approaches are quiet different. Classical criteria coming from decision theory only take into account extreme scenario. The methodology introduced by Bertsimas and Sim represents a way to qualify worst case analysis by considering that only a subset of coefficients will vary in the bad sense. In the approach coming from the decision theory, a solution is robust when it optimizes a particular criterion. In the approach coming from multicriteria analysis, the concept of robustness is not characterized anymore by optimality on a single criterion, but by a list of principles and conditions that leads to determine a set of robust solutions (this set being able to be empty!).

\section{Complexity and resolution of robust shortest path pro- blems in the interval model}

\subsection{With the worst case criterion}

This version of the robust shortest path problem, noted ROBINTWOR, is studied in [13]. Let us recall that, in this version, a path $\mu$ has a value $v_{\mathrm{wOR}}(\mu)=\max _{s \in S} c^{s}(\mu)$ and the problem is to determine the optimal path that minimizes this value on all paths. Given a path $\mu$ from 1 to $n$, the scenario which maximizes $c^{s}(\mu)$ is the one for which each arc $u \in U$ has the higher possible value, that is to say $B_{u}$. Since this worst case scenario, noted $s^{B}$, is unique, it is enough to consider the graph evaluated by this worst case scenario, and to determine the shortest path which exactly corresponds to $\mu_{\mathrm{wOR}}^{*}$ since $v_{\mathrm{wOR}}\left(\mu_{\mathrm{wOR}}^{*}\right)=\min _{\mu \in \mathcal{C}} B(\mu)$. Consequently,

THEOREM. [13] The ROBINTWOR problem is polynomial.

EXAMPLE. In the graph of the figure 1, the optimal path according to the worst case criterion is $\mu_{\mathrm{wOR}}^{*}=(1,2,6,7)=\mu_{1}$ of value 19 .

\subsection{With the maximum regret criterion}

This version of the robust shortest path problem, denoted ROBINTREG, is studied in $[4,13,17,18,19,28]$. Most results are based on the following property, established by Karaşan, Pinar and Yaman. Let us first recall that, in this problem version, a path $\mu$ has the value $v_{\mathrm{REG}}(\mu)=\max _{s \in S} r^{s}(\mu)=\max _{s \in S}\left(c^{s}(\mu)-c^{s}\left(\mu_{G^{s}}^{*}\right)\right)$.

Propriété 1 [13] Given a path $\mu$ from 1 to $n$, the scenario, denoted $s(\mu)$, which maximizes $r^{s}(\mu)$ is the one for which each arc $(i, j)$ belonging to $\mu$ has a value equals to its 
higher possible value, in other words $c_{i j}^{s(\mu)}=B_{i j}$, and each arc $(k, h)$ not belonging to $\mu$ has a value equals to its lower bound, in other words $c_{k h}^{s(\mu)}=b_{k h}$.

The scenario $s(\mu)$ is called the scenario induced by $\mu$. The problem to determine the robust path, according to the maximum regret criterion, is to find $\mu_{\mathrm{REG}}^{*}$ such as :

$$
v_{\mathrm{REG}}\left(\mu_{\mathrm{REG}}^{*}\right)=\min _{\mu \in \mathcal{C}}\left(c^{s(\mu)}(\mu)-c^{s(\mu)}\left(\mu_{G^{s(\mu)}}^{*}\right)\right)
$$

EXAMPLE. For the graph of the figure 1, the table 4 presents the maximum regret value of each path.

\begin{tabular}{|c||c|c|c|}
\hline$\mu$ & $c^{s(\mu)}(\mu)$ & $c^{s(\mu)}\left(\mu_{G^{s(\mu)}}^{*}\right)$ & $v_{\mathrm{REG}}(\mu)$ \\
\hline \hline$\mu_{1}=(1,2,6,7)$ & 19 & 3 & 16 \\
$\mu_{2}=(1,2,6,5,7)$ & 24 & 6 & 18 \\
$\mu_{3}=(1,2,4,5,7)$ & 24 & 5 & 19 \\
$\mu_{4}=(1,2,4,3,5,7)$ & 26 & 5 & 21 \\
$\mu_{5}=(1,3,2,6,7)$ & 27 & 5 & 22 \\
$\mu_{6}=(1,3,2,4,5,7)$ & 32 & 5 & 27 \\
$\mu_{7}=(1,3,2,6,5,7)$ & 32 & 8 & 24 \\
$\mu_{8}=(1,3,5,7)$ & 20 & 5 & 15 \\
\hline
\end{tabular}

TAB. 4 - Maximum regret value of each path from 1 to 7

The path $\mu_{8}$ is optimal for the maximum regret criterion ( $\mu_{8}$ is also the optimal path for the best case criterion).

Unfortunately, the difficulty comes from the fact that the number of elementary paths from 1 to $n$ may be exponential.

\subsubsection{Complexity}

This problem was shown to be NP-hard for directed graphs by Zieliński in [28] with a reduction to a particular version of the partition problem known as being NP-hard (see on this subject [9]). In parallel, Averbakh and Lebedev established in [4] that ROBINTREG is strongly NP-hard for non-directed graphs, by making a reduction to the Hamiltonian path problem, denoted HAMIL. They notice that their result can be easily extended for the case of acyclic directed graphs with a layered structure. In the following, we present this extension. Let us first recall that a graph has a layered structure if it is possible to partition the vertices set $X$ into disjoint subsets $X_{1}, \ldots, X_{g}$, called blocks, such as each arc goes 
from a vertex of a block $X_{j}$ to a vertex of the next block $X_{j+1}$.

THEOREM. [4] The problem ROBINTREG is NP-hard even for the acyclic directed graphs with a layered structure.

To prove this theorem, we have to introduce the decision version of the problem ROBINTREG which is defined by :

Instance : an acyclic directed graph $G=(X, U)$ with a layered structure, having two particular vertices 1 and $n$, whose $\operatorname{arcs} u \in U$ are valued by intervals $\left[b_{u}, B_{u}\right]$ and an integer $M$,

Question : does there exist a path from 1 to $n$ in $G$ with a maximum regret value lower or equal to $M$ ?

The HAMIL problem is defined as follows :

Instance : a non-directed connected graph $F=(V, E)$.

Question : does there exist in $F$ an Hamiltonian chain, that is to say a chain containing each vertex only once?

An instance $F=(V, E)$ of this problem, with $|V|=p$, can be polynomially reduced to an instance of the decision problem ROBINTREG in the following way. Vertices of $G$ are vertices $1, n$ and $2 p$ copies of the set $V$. The copy numbered $i$, denoted $V_{i}$, is the block $i$ of $G$. For any vertex $v \in V$, we note $v_{i}$ its copy in the block $i$, for $i=1, \ldots, 2 p$. Thus, we have $X=\left(1, V_{1}, \ldots, V_{2 p}, n\right)$, with $|X|=2+2 p^{2}$. The set $U$ of $G$ is defined as follows :

$-\operatorname{arcs}(1, v)$, for any vertex $v \in V_{1}$,

- $\operatorname{arcs}(v, n)$, for any vertex $v \in V_{2 p}$,

- $\operatorname{arcs}\left(v_{i}, v_{i+1}\right)$, for any vertex $v \in V$ with $i=1, \ldots 2 p-1$, these arcs will be called horizontal arcs,

$-\operatorname{arcs}\left(v_{2 i}, w_{2 i+1}\right)$ and $\left(w_{2 i}, v_{2 i+1}\right)$, for all $i=1, \ldots p-1$ and for all $\operatorname{arcs}(v, w)$ belonging to $E$.

The set of horizontal arcs is noted $H$. Any arc of $U$ which is not horizontal is called diagonal. The set of diagonal arcs is denoted $D$, among which we distinguish the subset $\tilde{D}$ containing arcs of $D$ not linked to 1 nor $n$. Intervals associated to horizontal arcs are $[0,1]$, and those associated to diagonal arcs are $[1,1]$.

In the figure 3, an example of graph $F$ with 3 vertices is represented on the left and the graph $G$ corresponding to the reduction is given on its right (in which intervals $[1,1]$ are replacing by the value 1). Let us observe that the vertices of the even blocks $V_{2 i}$, $i=1, \ldots, p$ can be reached by some vertices of the block $V_{2 i-1}$ only with horizontal arcs. For any vertex $v \in V$, the path $\left(1, v_{1}, \ldots, v_{2 p}, n\right)$ is called a $v$-tunnel. 


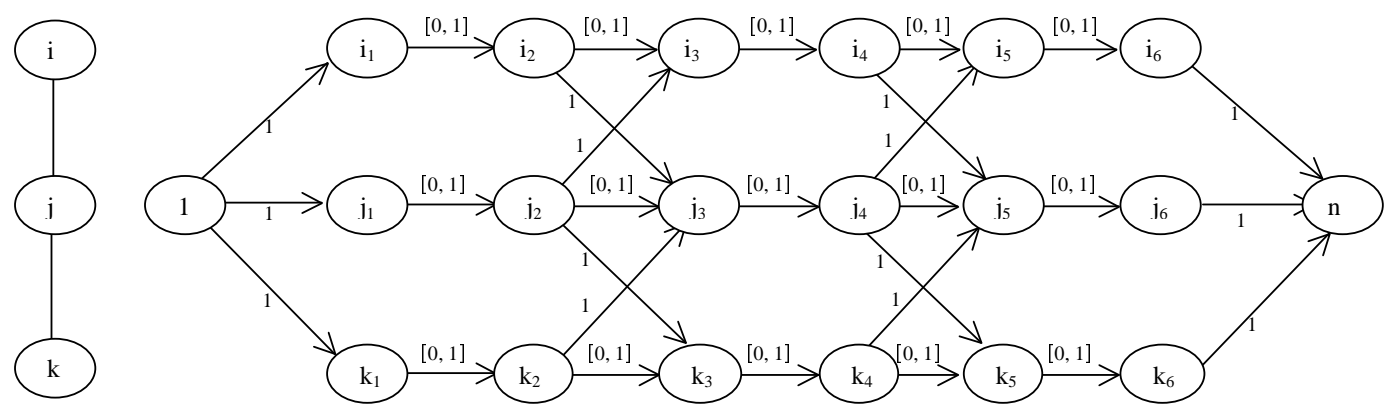

FIG. 3 - Example of the reduction of Hamiltonian path problem to ROBINTREG problem

It should be shown that an instance of HAMIL problem has a positive answer if and only if the corresponding instance of ROBINTREG problem has a positive answer for $M=$ $2 p-2$.

For a path $\mu$ from 1 to $n$, we denote $|\mu|$ its number of arcs and $\bar{\mu}$ its vertices belonging to $V_{1}, \ldots, V_{2 p}$. By definition of scenario induced by $\mu$ and according to upper bounds on arcs of the graph $G$, we have : $c^{s(\mu)}(\mu)=|\mu|=2 p+1$. Moreover under scenario $s(\mu)$, any arc of $D$ and any arc of $H$ which belongs to $\mu$ has a unit cost, whereas the cost of any arc of $H$ which does not belong to $\mu$ is equal to 0 . Consequently, the cost of a path $\lambda$ from 1 to $n$ in $s(\mu)$ is : $c^{s(\mu)}(\lambda)=|D \cap \lambda|+|H \cap \lambda \cap \mu|=2+|\tilde{D} \cap \lambda|+|H \cap \lambda \cap \mu|$. With $f(\mu, \lambda)=c^{s(\mu)}(\mu)-c^{s(\mu)}(\lambda)$, we have :

$$
f(\mu, \lambda)=2 p-1-|\tilde{D} \cap \lambda|-|H \cap \lambda \cap \mu|
$$

Let us suppose that there exists an Hamiltonian chain in $F$ denoted $\left(v^{1}, \ldots, v^{p}\right)$. Then, the sequence $\left(1, v_{1}^{1}, v_{2}^{1}, v_{3}^{2}, v_{4}^{2}, \ldots, v_{2 p-1}^{p}, v_{2 p}^{p}, n\right)$ is a path $\mu$ from 1 to $n$ in $G$. Since each tunnel $T$ does not have any arc in $\tilde{D}$ and exactly one horizontal arc in common with $\mu$, we have $f(\mu, T)=2 p-2$. Moreover, since any path $\lambda$ from 1 to $n$ which is not a tunnel has at least one arc in $\tilde{D}$, we have $: f(\mu, \lambda) \leq 2 p-2$. Consequently,

$$
v_{\mathrm{REG}}(\mu)=\max _{\lambda \in \mathcal{C}} f(\mu, \lambda)=2 p-2
$$

Let us now consider an optimal path $\mu$ from 1 to $n$ whose maximum regret is lower or equal to $2 p-2 . T$ is a tunnel such that $k=|H \cap T \cap \mu|=|\bar{T} \cap \bar{\mu}|$ is minimum. Since $\mu$ is optimal with a value lower or equal to $2 p-2$, and according to the equation 1 , we have : $f(\mu, T)=2 p-1-k \leq 2 p-2$. This implies $k \geq 1$. Thus each tunnel intersects $\bar{\mu}$ with at least $k$ different horizontal arcs, and one needs at least $p-1$ diagonal arcs in $\bar{\mu}$ to 
connect together these $k$ arcs. So, we have : $|\bar{\mu}|=2 p-1 \geq k p+p-1$ implying $k \leq 1$. Consequently, $k=1, f(\mu, T)=2 p-2$ and $v_{\mathrm{REG}}(\mu)=2 p-2$ with exactly one horizontal arc of the form $\left(v_{j}, v_{j+1}\right) \forall v \in V$ and exactly one diagonal arc of the form $\left(v_{2 i}, w_{2 i+1}\right)$ $\forall I=1, \ldots, p-1$ in $\mu$. Finally, we obtain an Hamiltonian chain in $F$ from the diagonal $\operatorname{arcs}$ of $\bar{\mu}$.

\subsubsection{Resolution}

Several algorithms for exactly solving ROBINTREG have been proposed [13, 17, 18, 19]. In these studies, ROBINTREG is written as an integer linear program with variables $y_{i j}$ which represent a path $\mu$ as follows :

$$
y_{i j}= \begin{cases}1 & \text { if }(i, j) \in \mu \\ 0 & \text { otherwise }\end{cases}
$$

The integer linear program, denoted $\mathcal{P}^{0}$, is :

$$
\mathcal{P}^{0} \begin{cases}\min & \max _{s \in S}\left(\sum_{(i, j) \in U} c_{i j}^{s} y_{i j}-x^{s}\right) \\
\text { s.c. } & \sum_{(i, j) \in U} y_{i j}-\sum_{(k, i) \in U} y_{k i}=\left\{\begin{array}{ll}
1 & \text { if } i=1 \\
-1 & \text { if } i=n \\
0 & \text { otherwise } \\
& y_{i j} \in\{0,1\} \forall(i, j) \in U
\end{array} \quad \forall i \in X\right.\end{cases}
$$

where $x^{s}$ is the length of the shortest path from 1 to $n$ in $G^{s}$.

According to the property 1 and given a path $\mu$ represented by $y$, the scenario which carries out $\max _{s \in S} \sum_{(i, j) \in U} c_{i j}^{s} y_{i j}-x^{s}$ is the induced scenario $s(\mu)$. Under this scenario, the length of any arc $(i, j)$ can be written in function of $y_{i j}$ as follows : $b_{i j}+\left(B_{i j}-b_{i j}\right) y_{i j}$. Thus, by introducing variables $x_{i}$ which represent the length of the shortest path from 1 to $i$ under scenario $s(\mu)$, Karaşan et al. in [13] propose a new formulation of ROBINTREG, noted $\mathcal{P}^{1}$

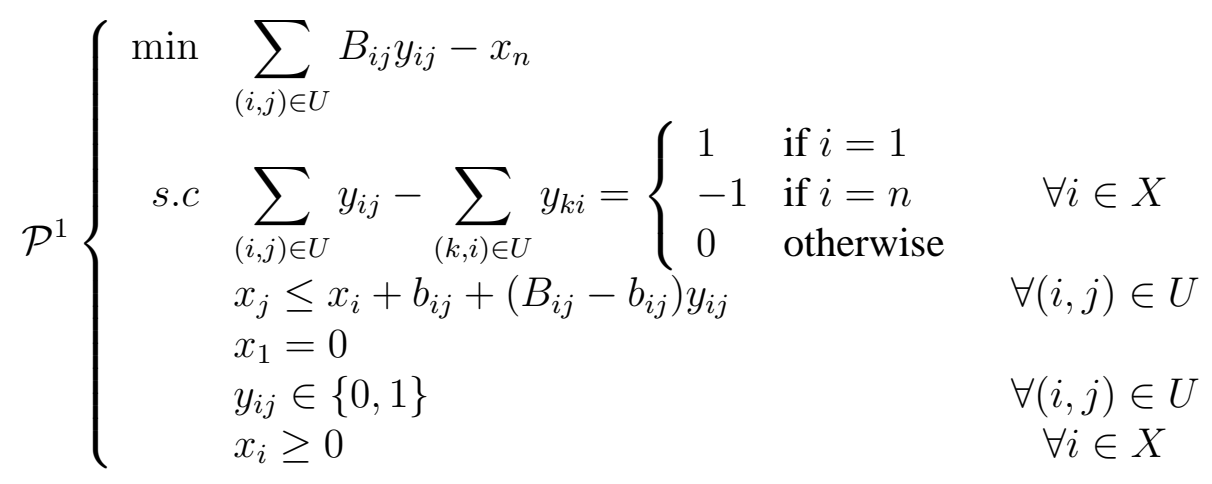


$\mathcal{P}^{1}$ is thus a mixed linear program. Karaşan et al. in [13] solve it using a linear programming solver. However, when $G$ is of big size, $\mathcal{P}^{1}$ cannot be solved exactly. The difficulty comes from the constraints $(2)$ which, if deleated, would reduce $\mathcal{P}^{1}$ to a simple shortest path problem. Also, in [13], authors propose to apply a preprocessing procedure which removes some constraints (2), those associated with some arcs that cannot belong to the optimal path. By reducing the size of $\mathcal{P}^{1}$, this procedure makes possible to increase the size of exactly solved problems.

More recently, Montemanni et al. proposed two algorithms to solve exactly the problem : a dedicated algorithm presented in [17], and a branch and bound algorithm presented in [19]. In [18], authors propose to apply a Benders decomposition scheme on $\mathcal{P}^{1}$. A great number of experiments is carried out to compare these various approaches. It seems that the algorithm resulting from the Benders decomposition is most powerful to solve most of the considered problems.

\subsection{With the multicriteria analysis methodology}

In the interval model, a path $\mu$ dominates a path $\mu^{\prime}$, denoted by $\mu \Delta \mu^{\prime}$, either if $\delta^{*}<0$ with $\delta^{*}=\max _{s \in S} c^{s}(\mu)-c^{s}\left(\mu^{\prime}\right)$, or $\left[\delta^{*}=0\right.$ and $\left.\exists s \in S: c^{s}(\mu)<c^{s}\left(\mu^{\prime}\right)\right]$. The complexity of this dominance test comes from the infinite set of scenarios to be considered. Dias and Climaco studied this problem in [8] for a more general model (for which the interval model is a particular case). They introduced binary relations to compare path evaluation vectors on the basis of two extreme scenarios only, the best case and the worst case scenarios (already introduced in section 4.1). In the best case scenario, denoted $s^{b}$, the value of each arc $u \in U$ is equal to the smallest cost, that is to say $c_{u}^{s^{b}}=b_{u}$. They defined the following binary relations :

Relation 1: $\mu \ll \mu^{\prime}$ if and only if $c^{s^{B}}(\mu)<c^{s^{b}}\left(\mu^{\prime}\right)$

Relation 2 : $\mu<\mu^{\prime}$ if and only if $\left[c^{s^{b}}(\mu)=c^{s^{b}}\left(\mu^{\prime}\right)\right.$ and $\left.c^{s^{B}}(\mu)<c^{s^{B}}\left(\mu^{\prime}\right)\right]$, or $\left[c^{s^{b}}(\mu)<\right.$ $c^{s^{b}}\left(\mu^{\prime}\right)$ and $\left.c^{s^{B}}(\mu)=c^{s^{B}}\left(\mu^{\prime}\right)\right]$

Relation 3 : $\mu<\approx \mu^{\prime}$ if and only if $c^{s^{b}}(\mu) \leq c^{s^{b}}\left(\mu^{\prime}\right)$ and $c^{s^{B}}(\mu) \leq c^{s^{B}}\left(\mu^{\prime}\right)$

On the basis of these three relations, they established the following results in the comparison of two paths $\mu$ and $\mu^{\prime}$ of $\mathcal{C}$ :

$\mu \ll \mu^{\prime} \Rightarrow \mu \Delta \mu^{\prime}$.

$c^{s^{b}}\left(\mu^{\prime}\right)<c^{s^{b}}(\mu) \Rightarrow \mu$ cannot dominate $\mu^{\prime}$.

$c^{s^{B}}\left(\mu^{\prime}\right)<c^{s^{B}}(\mu) \Rightarrow \mu$ cannot dominate $\mu^{\prime}$.

$\mu \Delta \mu^{\prime} \Rightarrow \mu<\approx \mu^{\prime}$, with the corollary : if $\mu$ is not $<\approx$-dominated then $\mu$ is not dominated.

From these results, they proposed an algorithm, based on the enumeration of $k$ shortest paths, to determine the set of non-dominated paths. In this algorithm, paths are enumerated in ascending order of their value on the scenario $s^{b}$, then $<\approx$-dominated and $\ll-$ dominated paths are removed, and finally, the complete dominance test is carried out only 
on remaining paths in order to eliminate the dominated ones.

In the specific case of interval model, the complete dominance test has not to be carried out. The property below allows to conclude by considering only the extreme best and worst case scenarios. Let us first denote by $\mu \backslash \mu^{\prime}$ the set of arcs that belong to $\mu$ and not to $\mu^{\prime}$.

Propriété $2 \mu$ dominate $\mu^{\prime}$ if and only if $c^{s^{B}}\left(\mu \backslash \mu^{\prime}\right) \leq c^{s^{b}}\left(\mu^{\prime} \backslash \mu\right)$.

Proof. Let us show that $c^{s^{B}}\left(\mu \backslash \mu^{\prime}\right) \leq c^{s^{b}}\left(\mu^{\prime} \backslash \mu\right) \Rightarrow \mu \Delta \mu^{\prime}$.

$c^{s^{B}}\left(\mu \backslash \mu^{\prime}\right) \geq c^{s}\left(\mu \backslash \mu^{\prime}\right), \forall s \in S$ and $c^{s^{b}}\left(\mu^{\prime} \backslash \mu\right) \leq c^{s}\left(\mu^{\prime} \backslash \mu\right), \forall s \in S$. Consequently, $c^{s^{B}}\left(\mu \backslash \mu^{\prime}\right) \leq c^{s^{b}}\left(\mu^{\prime} \backslash \mu\right)$ implies :

$$
\begin{aligned}
& \Rightarrow c^{s}\left(\mu \backslash \mu^{\prime}\right) \leq c^{s}\left(\mu^{\prime} \backslash \mu\right), \forall s \in S \\
& \Leftrightarrow c^{s}\left(\mu \backslash \mu^{\prime}\right)+\sum_{u \in \mu \cap \mu^{\prime}} c_{u}^{s} \leq c^{s}\left(\mu^{\prime} \backslash \mu\right)+\sum_{u \in \mu^{\prime} \cap \mu} c_{u}^{s}, \forall s \in S \\
& \Leftrightarrow c^{s}(\mu) \leq c^{s}\left(\mu^{\prime}\right), \forall s \in S
\end{aligned}
$$

Let us show now that $\mu \Delta \mu^{\prime} \Rightarrow c^{s^{B}}\left(\mu \backslash \mu^{\prime}\right) \leq c^{s^{b}}\left(\mu^{\prime} \backslash \mu\right)$.

Under scenario $s(\mu), c^{s(\mu)}(\mu)=c^{s^{B}}(\mu)$ and $c^{s(\mu)}\left(\mu^{\prime}\right)=c^{s^{b}}\left(\mu^{\prime}\right)+\sum_{u \in \mu^{\prime} \cap \mu}\left(B_{u}-b_{u}\right)$. But, with the dominance property of $\mu$ for the specific scenario $s(\mu)$, we obtain :

$$
\begin{aligned}
c^{s^{B}}(\mu) & \leq c^{s^{b}}\left(\mu^{\prime}\right)+\sum_{u \in \mu^{\prime} \cap \mu}\left(B_{u}-b_{u}\right) \\
\Leftrightarrow c^{s^{B}}\left(\mu \backslash \mu^{\prime}\right) & \leq c^{s^{b}}\left(\mu^{\prime} \backslash \mu\right)
\end{aligned}
$$

REMARK. If $\mu$ and $\mu^{\prime}$ have not any joint arc, then the property 2 becomes : $\mu \Delta \mu^{\prime} \Leftrightarrow$ $c^{s^{B}}(\mu) \leq c^{s^{b}}\left(\mu^{\prime}\right)$.

EXAMPLE. In the graph represented in the figure $4, \mu=(a, b, d, e)$ dominates $\mu^{\prime}=$ $(a, c, d, e)$. And, it is checked that $c^{s^{B}}\left(\mu \backslash \mu^{\prime}\right)=5<c^{s^{b}}\left(\mu^{\prime} \backslash \mu\right)=6$ with $c^{s^{B}}(\mu)=11>$ $c^{s^{b}}\left(\mu^{\prime}\right)=10$.

From the property 2, it is possible to propose an algorithm, similar to the one proposed by Dias and Climaco in [8], based on the enumeration of the $k$ shortest paths. Ones have to enumerate paths in ascending order of their value on the best case scenario and, in the event of equality, by ascending order of their value on the worst case scenario. Consequently, the ith enumerated path, denoted $\mu_{i}$, cannot be dominated by $\mu_{k}$ if $i<k$. On the other hand, $\mu_{i}$ dominates $\mu_{k}$ if and only if $c^{s^{B}}\left(\mu_{i} \backslash \mu_{k}\right) \leq c^{s^{b}}\left(\mu_{k} \backslash \mu_{i}\right)$. This test is the single test to be carried out for each enumerated path. 


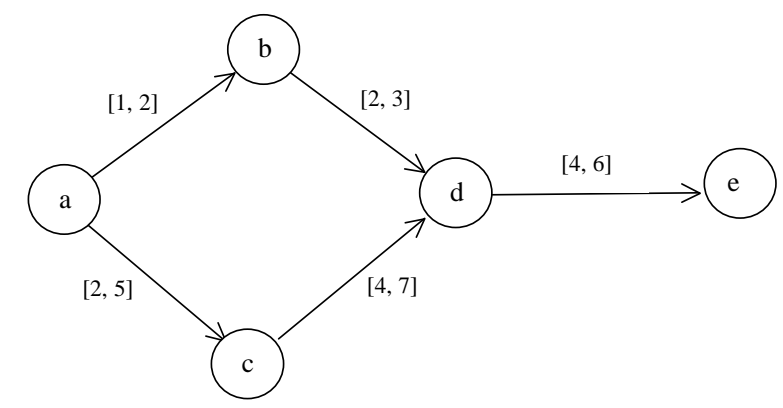

FIG. 4 - Dominance test for graph with intervals

\subsection{With the mathematical programming methodology}

In [5], authors show that the problem $\mathcal{P}_{\text {ROB }}$ presented in section 3.2 can easily be solved by solving at most $m+1$ shortest path problems. In fact, the optimal solution value of $\mathcal{P}_{\mathrm{ROB}}$, denoted $Z^{\star}$ is given by :

$$
Z^{\star}=\min _{k=1, \ldots m} Z^{k}
$$

where

$$
Z^{k}=\Gamma \widehat{c}_{k}+\min _{y \in Y}\left(\sum_{(i, j) \in U} \bar{c}_{i j} y_{i j}+\sum_{h=1}^{k}\left(\widehat{c}_{h}-\widehat{c}_{k}\right) y_{h}\right)
$$

where it is assumed that the indices of the arcs are ordered such that $\widehat{c}_{1} \geq \widehat{c}_{2} \geq \ldots \widehat{c}_{m}$.

Consequently, in this approach, the robust version presents the same complexity as the nominal one. Thus, in the case of the shortest path problem, the robust version can be polynomially solved which makes this approach very attractive.

\section{Complexity and resolution of robust shortest path pro- blems in the discrete set of scenarios model}

\subsection{With the worst case criterion}

\subsubsection{Complexity}

This version of the robust path problem, denoted ROBDISWOR, is studied in [15, 27]. Yu and Yang show that this problem is weakly NP-difficult if the number of scenarios is 
bounded by a constant and strongly NP-difficult otherwise. Indeed, they show in [27] that the 2-partition problem

Instance : a set $I$ of $m$ elements and a size $a_{i} \in \mathbb{Z}_{+}$for each $i$ in $I$.

Question : Does there exist a sub-set $I^{\prime} \subseteq I$ such as $\sum_{i \in I^{\prime}} a_{i}=\sum_{i \in I \backslash I^{\prime}} a_{i}$ ?

is reduced to ROBDISWOR problem defined as follows :

Instance : a connected graph $G=(X, U)$ with 2 scenarios.

Question : Does there exist a path $\mu$ from 1 to $n$ such as $v_{\text {WOR }}(\mu)=v$ ?

For that, we only have to consider the graph $G=(X, U)$ reduced to the 2-partition problem (represented in the figure 5) without directed cycle valued by two scenarios. This graph presents $2 m+2$ levels with $X=X_{0} \cup X_{1} \cup \ldots \cup X_{2 m+1}$ where $X_{0}=\{1\}$, $X_{2 m+1}=\{n\}$ (with $n=4 m+2$ ) and $X_{i}=\left\{x_{i, 1}, x_{i, 2}\right\}$. There are three categories of arcs in $U$ defined as follows :

- $U_{1}=\left\{\left(1, x_{1,1}\right),\left(1, x_{1,2}\right),\left(x_{2 m, 1}, n\right),\left(x_{2 m, 2}, n\right)\right\}$, each arc $(i, j)$ in $U_{1}$ is valued by $\{0,0\}$

- $U_{2}=\bigcup_{k=1}^{m-1}\left\{\left(x_{2 k, i}, x_{2 k+1, j}\right) / i=1,2 ; j=1,2\right\}$, each arc $(i, j)$ in $U_{2}$ is also valued by $\{0,0\}$

- $U_{3}=\left\{\left(x_{2 k-1, i}, x_{2 k, i}\right) / i=1,2 ; k=1, \ldots, m\right\}$, each arc in $U_{3}$ is valued in a different way :
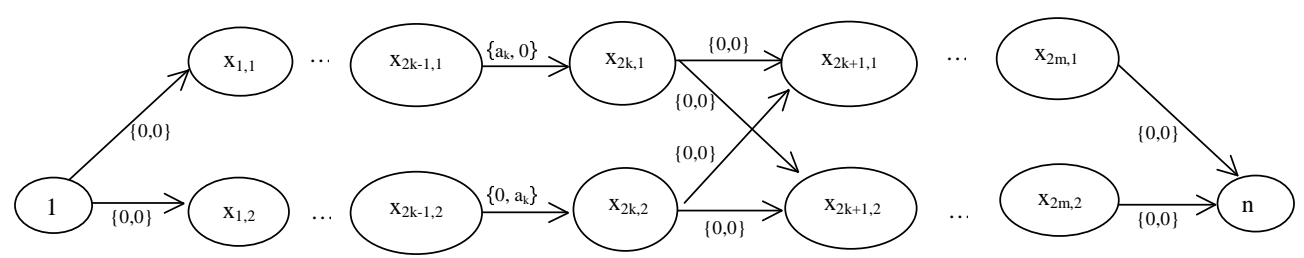

FIG. 5 - Reduction of the 2-partition problem to the ROBDISWOR problem

Considering a path $\mu$ from 1 to $n$ in $G$, let us set :

$$
\begin{aligned}
& I^{\prime}=\left\{k /\left(x_{2 k-1,1}, x_{2 k, 1}\right) \in \mu \forall k=1, \ldots, m\right\} \\
& I \backslash I^{\prime}=\left\{k /\left(x_{2 k-1,2}, x_{2 k, 2}\right) \in \mu \forall k=1, \ldots, m\right\}
\end{aligned}
$$

Thus $c^{1}(\mu)=\sum_{k \in I^{\prime}} a_{k}$ and $c^{2}(\mu)=\sum_{k \in I \backslash I^{\prime}} a_{k}$. Consequently, a 2-partition exists if and only if there exists a path $\mu$ from 1 to $n$ in $G$ such as $v_{\text {PIR }}(\mu)=\max \left\{c^{1}(\mu), c^{2}(\mu)\right\}=$ $\frac{\sum_{k \in I} a_{i}}{2}$, this value being the optimal path value $\mu_{\mathrm{PIR}}^{*}$. 


\subsubsection{Resolution}

$\mathrm{Yu}$ and Yang in [27] propose an exact algorithm for solving ROBDISWOR when the number of scenarios is bounded. It is based on the dynamic programming principle with a pseudo-polynomial time complexity. In particular for layered graphs, the complexity is $O\left(|U|\left(L_{\max }\right)^{q}\right)$ where $L_{\max }=\max _{s \in S} L_{s}$ with $L_{s}$ denoted the length of the longest path from 1 to $n$ for the scenario $s$. Yu and Yang also propose in [27] an approximate algorithm, denoted $H$. For layered graphs, the complexity is $O(|U| q)$. This algorithm consists to compute the shortest path for each scenario and, for a fictitious scenario which is an average of the $q$ scenarios, to determine the path which minimizes the worst case criterion. Given $\mu_{\mathrm{wOR}}^{h}$ the path determined by $H$, the authors show that :

$$
\frac{v_{\mathrm{WOR}\left(\mu_{\mathrm{WOR}}^{H}\right)}}{v_{\mathrm{WOR}\left(\mu_{\mathrm{WOR}}^{*}\right)}^{*}} \leq \frac{q \frac{\max _{s \in S} c^{s}\left(\mu_{\mathrm{WOR}}^{H}\right)}{\min _{s \in S} c^{s}\left(\mu_{\mathrm{WOR}}^{H}\right)}}{\frac{\max _{s \in S} c^{s}\left(\mu_{\mathrm{WOR}}^{H}\right)}{\min _{s \in S} c^{s}\left(\mu_{\mathrm{WOR}}^{H}\right)}+q-1}
$$

\subsection{With the maximum regret criterion}

Yu and Yang in [27] show that the 2-partition problem is reduced to ROBDISREG, for which the problem is to decide if there is a path $\mu$ from 1 to $n$ such as $v_{\mathrm{REG}}(\mu)=v$. The reduction presented in the previous section remains valid since $v_{\mathrm{REG}}(\mu)=v_{\mathrm{WOR}}(\mu)$ because $c^{1}\left(\mu_{G^{1}}^{*}\right)=c^{2}\left(\mu_{G^{2}}^{*}\right)=0$. In addition, exact and approximate algorithms for solving ROBDISWOR can be applied, after some minor modifications for solving ROBDISREG.

\subsection{With the multicriteria methodology}

In the discrete set of scenarios model, the problem of determining all non-dominated paths can be solved by applying algorithms defined in multicriteria analysis as suggested in [8]. Two types of algorithms are proposed in the literature for determining all nondominated paths in a multicriteria graph : graph algorithms which generalize classical monocriterion graph algorithms to the multicriteria case and, those based on the enumeration of the $k$ shortest paths. The efficiency of this approach rapidly decreases when the number of scenarios increases since the number of non-dominated paths significantly increases with the number of scenarios.

Concerning the problem to determine all Lorenz non-dominated paths, Perny and Spanjaard propose in [21] an $A^{*}$ algorithm. 


\section{Conclusion}

Robust path problem has been extensively studied. For most of the considered models, robust version of the shortest path problem becomes NP-difficult, excepted for the worst case analysis with the interval model which is tractable in polynomial time. However, the worst case analysis supposes that the scenario that will be realized will adversely affect the solution. This hypothesis may not be relevant in some decision context. In such cases, new models of robustness have to be proposed.

Concerning the modeling of uncertain, undetermined and random factors, it can be relevant in some context to consider them on graph structure and not only on arcs' value. In such a model, a scenario may be a particular partial subgraph. Such a "structural" model can be seen as an extension of probabilistic combinatorial optimization (cf [20] for the study of the longest probabilistic path).

\section{References}

[1] H. Aissi, C. Bazgan, and D. Vanderpooten. Complexity of the min-max and minmax regret assignment problems. Operations Research Letters, 33(6):634-640, 2005.

[2] I. Averbakh. Minmax regret solutions for minimax optimization problems with uncertainty. Operations Research Letters, 27:57-65, 2000.

[3] I. Averbakh. On the complexity of a class of combinatorial optimization problems with uncertainty. Math. Program., Ser. A, 90:263-272, 2001.

[4] I. Averbakh and V. Lebedev. Interval data minmax regret network optimization problems. Discrete Applied Mathematics, 138:289-301, 2004.

[5] D. Bertsimas and M. Sim. Robust discrete optimization and network flows. Math. Program., Ser. B, 98:49-71, 2003.

[6] D. Bertsimas and M. Sim. The price of robustness. Operations Research, 52(1):3553, 2004.

[7] V. G. Deĭneko and G. J. Woeginger. On the robust assignment problem under a fixed number of cost scenarios. Operations Research Letters, 34(2):175-179, 2006.

[8] L. C. Dias and J. N. Clímaco. Shortest path problems with partial information: models and algorithms for detecting dominance. European Journal of Operational Research, 121:16-31, 2000. 
[9] M.RM Garey and D.S. Johnson. Computers and Intractability: A Guide to the Theory of NP-Completeness. W.H. Freeman and Co., San Francisco, 1979.

[10] P. Hansen. Bicriterion path problems. In Multicriteria Decision making. In G. Fandel and T. Gal (eds), 1980.

[11] R. Hites, Y. De Smet, N. Risse, M. Salazar-Neumann, and P. Vincke. About the applicability of mcda to some robustness problems. European Journal of Operational Research, 174:322-332, 2006.

[12] R. Kalai, M.A. Aloulou, P. Vallin, and D. Vanderpooten. Robust 1-median location problem on a tree. In Proceedings of the ORP3 meeting, Valencia, Espagne, September 2005.

[13] O. E. Karaşan, M. Ç. Pinar, and H. Yaman. The robust shortest path problem with interval data. Technical report, Bilkent University, 2001.

[14] A. Kasperski and P. Zieliński. The robust shortest path problem in series-parallel multidigraphs with interval data. Operations Research Letters, 34(1):69-76, 2006.

[15] P. Kouvelis and G. Yu. Robust dicrete optimization and its applications. Kluwer Academic Publishers, Boston, 1997.

[16] R Montemanni. A benders decomposition approach for the robust spanning tree problem with interval data. European Journal of Operational Research, 174(3):1479-1490, 2006.

[17] R. Montemanni and L.M. Gambardella. An exact algorithm for the robust shortest path problem with interval data. Computers and Operations Research, 31:16671680, 2004.

[18] R. Montemanni and L.M. Gambardella. The robust path problem with interval data via benders decomposition. 4OR, 3(4):315-328, 2005.

[19] R. Montemanni, L.M. Gambardella, and A.V. Donati. A branch and bound algorithm for the robust shortest path problem with interval data. Operations Research Letters, 32:225-232, 2004.

[20] C. Murat and V. Paschos. The probabilistic longest path problem. Networks, 33:207219, 1999.

[21] P. Perny and O. Spanjaard. An axiomatic approach to robustness in search problems with multiple scenarios. In Proceedings of the 19th conference on Uncertainty in Artificial Intelligence, pages 469-476, Acapulco, Mexico, 2003. 
[22] B. Roy. Méthodologie multicritère d'aide à la décision. Economica, Paris, 1985.

[23] B. Roy. Flexibilité et Robustesse en Ordonnancement, chapter A propos de robustesse en recherche Opérationnelle et Aide à la Décision. Hermès, Paris, 2004.

[24] O. Spanjaard. Exploitation de préférences non-classiques dans les problèmes combinatoires : modèles et algorithmes pour les graphes. Thèse de doctorat, Université Paris Dauphine - LAMSADE, 2003.

[25] P. Vincke. Robust solutions and methods in decision-aid. Journal of multi-criteria decision analysis, 8:181-187, 1999.

[26] H. Yaman, O. E. Karaşan, and M. Ç. Pinar. The robust spanning tree problem with interval data. Operations Research Letters, 29:31-40, 2001.

[27] G. Yu and J. Yang. On the robust shortest path problem. Computers and Operations Research, 25(6):457-468, 1998.

[28] P. Zieliński. The computational complexity of the relative robust shortest path problem with interval data. European Journal of Operational Research, 158:570-576, 2004. 